\title{
Migration and Visa Liberalisation of Third Countries in E.U.
}

\author{
Ervis Moçka \\ Law Department, Faculty of Human Science, "Ismail Qemali" University of Vlora, Albania \\ Email: ervismocka@gmail.com
}

\section{Doi:10.5901/ajis.2013.v2n8p499}

\begin{abstract}
Only a year after signing the agreements of visa liberalization with Serbia, Montenegro and Macedonia, the EU removed the borders even for Albania and Bosnia and Herzegovina. A category of people who meet certain requirements would benefit from this process and move freely in the EU area. The migration of the EU should be analyzed even although none of the treaties establishing the European Community provide specific rates on this policy. Art 8/A of the Single European Act provides the common market as "... a space without internal frontiers in which the free movement of goods, persons, services and capital...". "No border area" requires the cooperation of member states in reinforcing controls on external borders and coordination in migration policies. The cooperation began as an intergovernmental one until the ratification of Amsterdam Treaty where many sections of this collaboration passed in the first pillar. This paper aims to analyze the evolution of the EU policy in migration focusing on the current legislative framework after the ratification of the Lisbon Treaty.
\end{abstract}

Keywords: visa liberalization, free movement of persons, migration in the EU, Schengen agreement, no border area.

\section{Introduzione}

La liberalizzazione dei visti Schengen in Serbia, Montenegro e Macedonia è avvenuta nel dicembre 2009. Un anno dopo il 15 dicembre 2010 la liberalizzazione dei visti Schengen è valida anche per Albania e Bosnia Erzegovina. La liberalizzazione si traduce in uno snellimento sostanziale delle procedure burocratiche. Gli accordi conclusi con la Repubblica d'Albania, la Bosnia-Erzegovina, la Repubblica del Montenegro, l'ex Repubblica lugoslava di Macedonia e la Repubblica di Serbia mirano a facilitare il rilascio dei visti per soggiorni di breve durata ai cittadini di questi paesi per soggiorni di massimo 90 giorni per periodi di 180 giorni. Quando i cittadini di questi paesi partner richiedono un visto per soggiorni di breve durata, essi beneficiano di requisiti documentali semplificati per poter giustificare lo scopo del loro viaggio nell'Unione Europea. I negoziati per la conclusione di accordi sulla liberalizzazione dei visti si sono avviati subito dopo il vertice UE-Balcani occidentali svoltosi a Salonicco il 21 giugno 2003 ("Agenda di Salonicco"), in occasione del quale i partecipanti si sono accordati sul principio della liberalizzazione dei visti. Questo processo negoziale, parallelo alle discussioni sulla conclusione di accordi di riammissione, è sfociato l'8 novembre 2007 nell'adozione, da parte del Consiglio, di una decisione relativa a ciascuno dei paesi partner ${ }^{1}$, che ha sancito la conclusione di un accordo con ciascuno di essi, in base all'articolo 62 del trattato che istituisce la Comunità Europea (ora 77 TFUE) in combinato disposto con l'articolo 300 (ora 218 TFUE).

La politica della liberalizzazione dei visti rientra nelle misure previste dall'articolo 77, n. 2, TFUE necessarie per conseguire l'obiettivo dell'Unione di offrire "ai suoi cittadini uno spazio di libertà, sicurezza e giustizia senza frontiere interne, in cui sia assicurata la libera circolazione delle persone insieme a misure appropriate per quanto concerne I controlli alle frontiere esterne, l'asilo, l'immigrazione, la prevenzione della criminalità e la lotta contro quest'ultima" indicato dall'articolo 3, n. 2 TUE. La disciplina puntuale per realizzare tale obiettivo è stabilita nel Trattato sul

\footnotetext{
1 Decisione 2007/821/CE del Consiglio, dell'8 novembre 2007, relativa alla conclusione dell'accordo di facilitazione del rilascio dei visti tra la Comunità europea e la Repubblica d'Albania. Decisione 2007/822/CE del Consiglio, dell'8 novembre 2007, relativa alla conclusione dell'accordo di facilitazione del rilascio dei visti tra la Comunità europea e la Bosnia-Erzegovina. Decisione 2007/823/CE del Consiglio, dell'8 novembre 2007, relativa alla conclusione dell'accordo di facilitazione del rilascio dei visti tra la Comunità europea e la Repubblica del Montenegro. Decisione 2007/824/CE del Consiglio, dell'8 novembre 2007, relativa alla conclusione dell'accordo di facilitazione del rilascio dei visti tra la Comunità europea e l'ex Repubblica iugoslava di Macedonia. Decisione 2007/825/CE del Consiglio, dell'8 novembre 2007, relativa alla conclusione dell'accordo di facilitazione del rilascio dei visti tra la Comunità europea e la Repubblica di Serbia.
} 
Funzionamento dell'Unione Europea (art. 67-89 Parte Terza, Titolo V, ripartito in cinque Capi). II Capo due è dedicato alle Politiche relative ai controlli alle frontiere, all'asilo e all'immigrazione (art.77-80), politiche queste strettamente legate l'una con l'altra. Prima di analizzare le novità che introduce in tale materie il Trattato di Lisbona è neccessario riportare una breve evoluzione excursus del quadro giuridico.

\section{Excursus del quadro giuridico.}

I trattati istitutivi della Comunità, non contenevano disposizioni in materia d'immigrazione e asilo, la disciplina legata ai cittadini di Stati terzi non era considerata d'interesse comune, ma come una materia di competenza esclusiva concernendo problemi di sicurezza pubblica e quindi espressione fondamentale della sovranità statale. Anche la libera circolazione delle persone obiettivo previsto alla lettera c) dell'art. 3, fu intesa come limitata a coloro che possedevano la cittadinanza di uno Stato membro, nonostante la disposizione utilizzava il termine "persone" senza alcun riferimento alla nazionalità e quindi idonea a comprendere anche i cittadini degli Stati terzi ${ }^{2}$. Anche l'art. 48, che prevedeva la libera circolazione dei lavoratori, non conteneva nessun riferimento alla nazionalità ${ }^{3}$, ma fu intesa dalle istituzioni comunitarie come limitata ai soli lavoratori che possedevano la cittadinanza di uno degli Stati membrit. Nella Comunicazione del $1979^{5}$ la Commissione indicava nei problemi concernenti l'ammissione, il soggiorno, l'allontanamento e la parità di trattamento dei lavoratori migranti, i settori nei quali doveva realizzarsi l'armonizzazione in materia d'immigrazione, tuttavia i tentativi della Commissione per una armonizzazione in materia non produssero nessun successo. Invocando l'art. 118 come base giuridica nella Decisione n. 85/381/CEE ${ }^{6}$ la Commissione tentò di istituire un obbligo di comunicazione e concertazione sulle politiche migratorie degli Stati membri ${ }^{7}$. La Decisione fu impugnata da cinque Stati membri e annullata dalla Corte di Giustizia perché oltrepassava i limiti dei poteri assegnati alla Commissione dall'art. 118 TCEE ${ }^{8}$. La decisione della Corte nonostante accoglieva il ricorso di annullamento proposta dagli Stati, dall'altro lato poneva le basi per la creazione di una politica immigratoria a livello comunitario. Con la sua decisione la Corte riconoscendo alla Commissione la competenza di adottare atti di natura vincolante in materia d'immigrazione, toglieva l'esclusiva agli Stati in una materia delicata e da sempre ritenuta dagli Stati membri come di loro competenza esclusiva poiché strettamente inerente ai problemi di sicurezza pubblica ${ }^{9}$, e apriva così la strada per l'inizio di una politica comunitaria in materia.

L'Atto Unico del 1986 non portò novità in materia nonostante l'interpretazione data dalla Commissione alla libera circolazione delle persone prevista dall'art. 8A, necessario per la realizzazione del mercato interno. Secondo l'interpretazione della Commissione, la libertà di circolazione doveva essere riconosciuta a tutti gli individui, indipendentemente dalla nazionalità ${ }^{10}$. Nel suo Libro Bianco ${ }^{11}$, la Commissione vedeva come necessaria per realizzare il mercato interno, l'abolizione dei controlli alle frontiere interne con la conseguenza di un rafforzamento degli stessi alle

2 II Trattato originario nella lettera c) art. 3 prevedeva "l'eliminazione fra gli Stati membri degli ostacoli alla libera circolazione delle merci, delle persone, dei servizi e dei capitali LANG. A, "La politica comunitaria in materia di immigrazione", in DPCE, 2003, p. 698.

3 Art. 48 par.1 "La libera circolazione dei lavoratori all'interno della Comunità è assicurata".

4 Così il Regolamento (CEE) n. 1612/68 relativo alla libera circolazione dei lavoratori all'interna della Comunità (in G.U. n. L. 257 del 19/10/1968 pag. 0002-0012) e il Regolamento (CEE) n. 1251/70 relativo al diritto dei lavoratori di rimanere sul territorio di uno Stato dopo aver occupato un impiego (in G.U. n. L 142 del 30/06/1970, p. 24), non usano l'espressione "lavoratori degli Stati membri" ma "cittadini di Stati membri che siano stati occupati in qualità di lavoratori dipendenti".

5 Comunicazione della Commissione al Consiglio su "La consultazione relativa alle politiche di migrazione nei confronti degli Stati terzi", COM (79) 115 def. del 27 marzo 1979.

${ }_{6}^{6}$ Decisione della Commissione dell'8 giugno 1988 che istituisce una procedura di comunicazione preliminare e di concertazione sulle politiche migratorie nei confronti dei paesi terzi, in GUCE, L 217 del 14 agosto 1985, p.25.

${ }^{7}$ Tale decisione faceva riferimento alle due Risoluzioni del Consiglio in materia dell'immigrazione, del 1976 e 19807, nonché a una Risoluzione del Parlamento Europeo del 9 giugno 1983 che invitava il Consiglio e la Commissione ad elaborare proposte "sull'armonizzazione delle politiche dei visti e della normativa riguardante i stranieri".

8 Sentenza della Corte di giustizia del 9 luglio 1987, cause riunite 281, 283, 284, 285 e 287/85,

Repubblica federale di Germania, Repubblica francese, Regno dei Paesi Bassi, Regno di Danimarca e Regno Unito di Gran Bretagna e d'Irlanda del Nord c. Commissione delle Comunità europee, in Racc. , 1987, p. 3203 ss.

9 Tale tesi sostenuta fortemente dalla Francia nella controversia in questione, vedi motivazione della sentenza, punto 9; sentenza completa consultabile anche sul sito: http://eur-law.eu/IT/Sentenza-Corte-9-Iuglio-1987-Repubblica-federale-Germania,146845,d

10 LIGUORI A., L'immigrazione e l'Unione Europea, in DCSI, 2000, p. 429

11 Libro Bianco: Il completamento del mercato interno, COM (1985) 310 del 14 giugno 1985, par.55. 
frontiere esterne, e da qui l'esigenza di politiche comuni in materia di visti, asilo e condizione dei cittadini paesi terzi e aveva indicato anche un calendario per la presentazione delle proposte.

\subsection{La Cooperazione intergovernativa:}

Gli Stati membri scelsero di sviluppare tra loro forme di cooperazione intergovernativa per l'attuazione della libera circolazione delle persone, piuttosto che coinvolgere le istituzioni comunitarie. Cinque Stati (Francia, Germania, Belgio, Lussemburgo, Paesi Bassi) conclusero tra loro l'Accordo di Schengen relativo all'eliminazione graduale dei controlli alle frontiere comuni e la relativa Convenzione d'Applicazione ${ }^{12}$ (successivamente firmati da tutti gli stati membri, ad eccezione del Regno Unito e Irlanda). Dall'abolizione dei controlli alle frontiere avrebbero approfittato non solo i cittadini comunitari ma anche i cittadini degli Stati terzi, per questa ragione gli articoli 7, 17 e 20 prevedevano l'impegno degli Stati da adottare delle misure relative alle politiche di visti, condizioni d'ingresso cittadini Stati terzi, diritto degli stranieri ecc. L'adozione delle misure previste nell'Accordo necessitava di un ulteriore azione degli Stati per essere tradotte in vere e proprie disposizioni specifiche, e quindi applicabili. Sono iniziate così delle lunghe trattative che hanno portato all'adozione della Convenzione dell'Applicazione dell'Accordo di Schengen, firmata il 19 giugno 1990, sempre nella città lussemburghese di Schengen. La Convenzione veniva suddivisa in otto Titoli. II Capitolo III regolava la disciplina dei visti. L'articolo 10 istituiva un visto uniforme, valido per tutto il territori Schengen per un periodo di soggiorno massimo di tre mesi. I visti per un soggiorno superiore a tre mesi erano considerati come visti nazionali, rilasciati dai singoli Stati in base ai propri ordinamenti e concedeva la possibilità al titolare di tale visto, di transitare nel territorio degli altri Stati contraenti della Convenzione. Per quanto riguardo l'ingresso nell'area Schengen degli cittadini degli Stati terzi per un soggiorno non superiore ai tre mesi, l'articolo 5 prevedeva che lo straniero doveva essere in possesso di: a) documenti validi; $b$ ) di un visto valido, se richiesto; c) doveva esibire se necessario documenti che giustificavano lo scopo e le condizioni del soggiorno, nonché disporre dei mezzi di sussistenza sufficienti, sia per la durata di soggiorno che per il ritorno nel paese di provenienza o per il transito verso uno Stato terzo; d) non doveva essere segnalato ai fini della non ammissione; e infine la lettera e) dell'articolo 5, prevedeva che lo straniero per poter entrare nell'area Schengen, non doveva essere considerato pericoloso per l'ordine pubblico, la sicurezza nazionale o le relazioni internazionali di uno degli Stati contraenti. I dati relativi agli stranieri ai fini di non ammissione erano inseriti in base ad una segnalazione nazionale in un sistema comune d'informazione denominato Sistema d'Informazione Schengen (SIS), previsto dall'art. 92.

\subsection{La nascita dell'Unione Europea e le politiche dell'immigrazione:}

II Trattato di Maastricht istituiva l'Unione Europea "fondata sulle Comunità europee, integrate dalle politiche e forme di cooperazione instaurate dal presente trattato". ${ }^{13}$ Veniva così costituita quella struttura che si basava in tre "pilastri": la Comunità, la Politica estera e di sicurezza comune e la Cooperazione in materia di giustizia e affari interni (GAl). L'articolo K.1 del TUE elencava come «questioni di interesse comune» tra altri, anche la politica di asilo, le norme sull'attraversamento delle frontiere esterne e la politica di immigrazione e la politica da seguire nei confronti dei cittadini dei paesi terzi, cioè le condizioni d'ingresso, circolazione e soggiorno dei cittadini di Stati terzi, le quali diventavano così, materie, parte di forme di cooperazione previste nell'ambito del terzo pilastro. Si trattava sempre di una forma di cooperazione intergovernativa, ma che si svolgeva nel quadro istituzionale dell'UE. Con il Trattato di Maastricht avvenne solo una comunitarizzazione della politica dei visti tramite l'inserimento dell'articolo $100 \mathrm{C}$, mentre il settore d'immigrazione veniva lasciato nell'ambito della cooperazione intergovernativa, cooperazione che veniva istituzionalizzato dal Trattato di Maastricht nell'ambito del c.d. "terzo pilastro". L'art. 100C prevedeva che il Consiglio deliberando all'unanimità ${ }^{14}$, su proposta della Commissione e previa consultazione del Parlamento Europeo, determinava un elenco dei paesi terzi cui cittadini devono essere in possesso di un visto per l'attraversamento delle frontiere esterne degli Stati membri e adottava le misure relative all'instaurazione di un modello uniforme di visto. Così il Consiglio usando

\footnotetext{
12 L'Accordo di Schengen del 14 giugno 1985 sulla graduale abolizione dei controlli alle frontiere interne e la Convezione d'Applicazione dell'Accordi di Schengen del 19 giugno 1990.

13 Art. A del Trattato di Maastricht.

${ }^{14}$ L'articolo K.9 nel n.3 prevedeva che dal $1^{\circ}$ gennaio 1996 il Consiglio avrebbe deliberato a maggioranza qualificata, e non più all'unanimità
} 
come base giuridica proprio l'articolo $100 \mathrm{C}$ ha adottato prima il Regolamento n. 1683/9515 il quale stabiliva un modello uniforme di visto, e poi il Regolamento n. $2317 / 95^{16}$ che determinava l'elenco dei paesi terzi i cui cittadini devono essere in possesso di un visto per l'attraversamento delle frontiere esterne degli Stati membri. Quest'ultimo Regolamento è stato annullato dalla Corte della Giustizia a causa della mancata consultazione del Parlamento Europeo nella procedura d'adozione ${ }^{17}$, ed è stato superato dal Regolamento n. 574/9918, a sua volta superato dal Regolamento 539/200119. Con l'entrata in vigore del Trattato di Amsterdam ${ }^{20}$ il $1^{\circ}$ Maggio 1999 lo scenario cambia completamente. Le misure relative all'immigrazione e cioè, l'ingresso, soggiorno e allontanamento dei cittadini di Stati terzi vengono inserite nel nuovo Titolo IV TCE intitolato «Visti, asilo, immigrazione e altre politiche connesse alla libera circolazione delle persone» e vengono così trasferite dal terzo al primo pilastro, divenendo materie di competenza comunitaria. Le misure previste dal Titolo IV vengono inserite nell'ambito di realizzazione di una finalità più generale che è: la creazione di "uno spazio di libertà, sicurezza e giustizia". Allo scopo di creare tale spazio l'articolo 61 TCE assegnava al Consiglio il potere di adottare: 1) "misure volte ad assicurare la libera circolazione delle persone in conformità all'articolo 14"; 2) "misure in materia di controlli alle frontiere esterne, asilo e immigrazione a norma degli articoli 62, punti 2 e 3 e dell'articolo 63 TCE .....Per quanto riguarda l'articolo 62 TCE questo assegna tre competenze alla Comunità: 1) competenza nell'abolizione dei controlli alle frontiere interne, 2) competenza nell'attraversamento delle frontiere esterne della Comunità, che prevedeva l'attuazione di misure cui gli Stati dovevano "attenersi per l'effettuazione di controlli sulle persone alle suddette frontiere" e "regole in materia di visti relativi a soggiorni non superiori a tre mesi21" e infine, 3) una competenza nella circolazione nell'area comunitaria degli cittadini Stati terzi per un periodo non superiore ai tre mesi. Per quanto riguarda l'articolo 63 il punto 3 riconosceva la competenza comunitaria nella materia d'immigrazione e particolarmente nei settori di: a) ingresso e soggiorno dei cittadini Stati terzi per periodi di lungo termine; b) ingresso e soggiorno irregolari e il rimpatrio di quest'ultimi. II punto 1) e 2) dell'articolo 63 invece assegnavano alla Comunità, competenze in materia d'asilo, rifugiati e sfollati. II Trattato di Amsterdam decide anche di integrare tramite un Protocollo ad hoc allegato al Trattato, l'acquis di Schengen nel diritto dell'Unione europea.

Con il Trattato di Lisbona è venuta a mancare la struttura in pilastri nella quale si fondava l'Unione fin dal momento della sua creazione e le decisioni sulla politica d'immigrazione vengono oramai adottate con procedura legislativa ordinaria. Nell'indicazione degli obiettivi dell'Unione individuati nell'art. 3 avviene un diverso ordine, dove il "mercato interno" cede il passo allo "spazio di liberta, sicurezza e giustizia". II conseguimento di tale spazio rientra nelle competenze concorrenti (art.4, n. 1, lett. J TFUE). All'Unione vengono conferiti poteri d'azione relativi per conseguire tale obiettivo. In base all'articolo 67, n. 2 l'Unione "garantisce che non vi siano controlli sulle persone alle frontiere interne e sviluppa una politica comune in materia di asilo, immigrazione e controllo delle frontiere esterne....", e tutto questo deve essere conseguito tramite le misure previste dall'articolo 77, n. 1, TFUE, quali: a) la politica comune dei visti e di altri titoli di soggiorno di breve durata; b) i controlli ai quali sono sottoposte le persone che attraversano le frontiere esterne; c) le condizioni alle quali i cittadini dei Paesi terzi possono circolare liberamente nell'Unione per un breve periodo; d) qualsiasi misura necessaria per l'istituzione progressiva di un sistema integrato di gestione delle frontiere esterne. I poteri d'azione

\footnotetext{
15 Regolamento (CE) n. 1683/95 del Consiglio del 29 maggio 1995, che istituisce un modello uniforme per i visti, in GUCE L 164 del 14 luglio 1995, questo è stato più volte modificato, modifiche introdotte dal Regolamento (CE) n. 334/2002 del Consiglio del 18 febbraio 2002 in GU L 53 p. 7 del 23 febbraio 2002, successivamente dal Regolamento (CE) n. 1791/2006 del Consiglio del 20 novembre 2006 in GU L 363 p.1 del 20 dicembre 2006 e poi dal Regolamento (CE) n. 856/2008 del Consiglio del 24 luglio 2008 in GU L 235 p.1 del 2 settembre 2008.

16 Regolamento (CE) n. 2317/95 del Consiglio del 25 settembre 1995, che determina quali siano i paesi terzi i cui cittadini devono essere in possesso di un visto per l'attraversamento delle frontiere esterne degli Stati membri, in GUCE L 234 del 3 ottobre 1995.

17 Sentenza della Corte di Giustizia del 10 giugno 1997, Parlamento c. Consiglio dell'Unione europea causa C 392/95, in Racc., 1, 1997, p. 3213 ss.

18 Regolamento (CE) n. 574/1999 del Consiglio del 12 marzo 1999 che determina quali siano i paesi terzi i cui cittadini devono essere in possesso di un visto per l'attraversamento delle frontiere esterne degli Stati membri in GU L 72 del 18.3.1999, pag. 2 ss.

19 Regolamento (CE) n. 539/2001 del Consiglio del 15 marzo 2001 che adotta l'elenco dei paesi terzi i cui cittadini devono essere in possesso del visto all'atto dell'attraversamento delle frontiere esterne e l'elenco dei paesi terzi i cui cittadini sono esenti da tale obbligo in GU L 81 del 21.3.2001, pag. 1. Tale regolamento ha subito otto ulteriori modifiche, di cui l'ultima dal Regolamento (UE) n. $1211 / 2010$ del Parlamento europeo e del Consiglio del 15 dicembre 2010 in GU L 339 del 22.12.2010, p. 6.

20 II testo del Trattato in GUCE C 340 del 10 novembre 1997.

21 Questa comprendeva l'adozione di "i) un elenco dei paesi terzi i cui cittadini devono essere in possesso del visto all'atto dell'attraversamento delle frontiere esterne e di quelli i cui cittadini sono esenti da tale obbligo; ii) le procedure e condizioni per il rilascio dei visti da parte degli Stati membri; iii) un modello uniforme di visto; iv) norme relative a un visto uniforme";
} 
riconosciuti all'Unione dal Trattato si traducono nell'adozione di atti normativi vincolanti che come prevede l'articolo 68 TFUE si devono mantenere entro gli orientamenti strategici della programmazione legislativa e operativa fissati dal Consiglio Europeo, il quale prima a Tampere (1999) e poi all'Aja (2004) ha adottato i Programmi che delinea le priorità dell'Unione Europea (UE) per lo spazio di libertà, sicurezza e giustizia per il periodo 2010-2014. II Programa di Stoccolma ${ }^{22}$ prevede uno sviluppo ulteriore delle politiche dell'UE sulla gestione integrata delle frontiere e in materia di visti per rendere l'accesso legale all'Europa più efficace per i cittadini non comunitari, accompagnata dal rafforzamento dei controlli delle frontiere e un sviluppo della politica comune in materia di visti.

\section{Fase d'attuazione.}

Sulla base del Patto Europeo sull'immigrazione e l'asilo del 2008, l'Unione Europea deve sviluppare una politica di migrazione globale e flessibile. Occorre anche rafforzare il dialogo e i partenariati con i paesi terzi (d'origine e di transito), in particolare tramite l'ulteriore sviluppo dell'approccio globale in materia di migrazione che prevede la conclusione, a livello comunitario o bilaterale, di accordi con i paesi di origine e di transito che comportino elementi concernenti la migrazione legale e clandestina, la riammissione nonché lo sviluppo di questi paesi. II Piano d'azione ${ }^{23}$ che fornisce la tabella di marcia per l'attuazione delle priorità politiche tracciate dal programma di Stoccolma per il periodo 2010 al 2014 prevede l'ulteriore sviluppo dell'approccio globale dell'Unione alla migrazione per accrescere la cooperazione con i paesi extraeuropei, come una delle azioni che la Commissione deve attuare per portare avanti la politica dell'immigrazione.

La politica dell'immigrazione nella fase attuale si realizza così in una dimensione globale entro un contesto di partenariato e cooperazione con i Paesi terzi. La liberalizzazione dei visti avviene proprio in questo nuovo contesto. L'Unione da parte sua sfruttando i poteri d'azione riconoscutosi dal Trattato ha datto la possibilita ai partner dei Balcani occidentali di concludere con essa accordi di facilitazione del rilascio dei visti in parallelo con la conclusione di accordi di riammissione. L'1 gennaio 2008 sono entrati in vigore gli accordi di facilitazione del visto con cinque paesi dei Balcani occidentali (Albania, Bosnia-Erzegovina, ex Repubblica iugoslava di Macedonia, Montenegro e Serbia), che costituiscono una prima tappa concreta del processo previsto dall'agenda di Salonicco verso un regime di esenzione dal visto per i cittadini dei paesi dei Balcani occidentali. Con ciascuno di questi paesi è stato avviato nel 2008 un dialogo sulla liberalizzazione dei visti, da realizzarsi mediante tabelle di marcia elaborate a tal fine. L'Unione con il Regolamento (CE) n. 810/2009 ha istituito un codice comunitario dei visti che fissa le procedure e le condizioni per il rilascio del visto per soggiorni di breve durata (non più di tre mesi su un periodo di sei mesi). II Regolamento che si applica dal 5 aprile 2010 ha modifica il regolamento VIS e il codice frontiere Schengen e ha abrogato inoltre gli articoli da 9 a17 della convenzione di applicazione dell'accordo di Schengen e le istruzioni consolari comuni. Dall'altra parte i paesi partner dei Balcani occidentali per approfittarne dalla politica della liberalizzazione dei visti dovevano soddisfare i parametri di riferimento predisposte nelle rispettive tabelle di marcia dalla Commissione. Tali parametri concernevano a settori quali la sicurezza dei documenti, gestione delle frontiere, asilo, migrazione, lotta alla criminalità organizzata e alla corruzione e protezione dei diritti fondamentali. Nelle sue considerazioni del 2009 la Commissione riteneva che i paesi partner avessero soddisfatto i parametri previsti nelle tabelle di marcia e proponeva cosi la liberalizzazione dei visti. Con il Regolamento (CE) n. 1244/2009 del Consiglio del 30 novembre 2009 e il Regolamento (UE) n. 1091/2010 del Parlamento Europeo e del Consiglio del 24 novembre 2010 che modificano il regolamento (CE) n. 539/2001 del Consiglio che adotta l'elenco dei paesi terzi i cui cittadini devono essere in possesso del visto all'atto dell'attraversamento delle frontiere esterne e l'elenco dei paesi terzi i cui cittadini sono esenti da tale obbligo veniva liberalizzato i visti con i Paesi partner dei Balcani occidentali.

Dal 19 dicembre 2009 i cittadini dell'ex Repubblica Jugoslava di Macedonia, del Montenegro e della Serbia, titolari di passaporto biometrico, possono viaggiare verso gli Stati membri dell'Unione Europea senza visto a norma del regolamento n. 539/2001. Alle medesime condizioni, i cittadini dell'Albania e della Bosnia-Erzegovina beneficiano della stessa libertà di viaggiare senza visto verso gli Stati membri dell'Unione dal 15 dicembre 2010.

\footnotetext{
22 Programma di Stoccolma - Un'Europa aperta e sicura al servizio e a tutela dei cittadini [Gazzetta ufficiale C 115 del 4.5.2010].

${ }^{23}$ Comunicazione della Commissione al Parlamento europeo, al Consiglio, al Comitato economico e sociale europeo e al Comitato delle regioni del 20 aprile 2010 - Creare uno spazio di libertà, sicurezza e giustizia per i cittadini europei - Piano d'azione per l'attuazione del programma di Stoccolma [COM(2010) 171 definitivo
} 


\section{Problematica, misure in atto e Raccomandazioni}

La liberalizzazione dei visti ai paesi dei Balcani occidentali, non è passata senza creare dei problemi specialmente per alcuni dei paesi membri dell'Unione quali la Germania, Svezia e Belgio che sono stati particolarmente interessati dall'aumento di richieste d'asilo per motivazioni economiche. Nel gennaio 2011 la Commissione europea avviò un meccanismo di monitoraggio successivo alla liberalizzazione dei visti che copre tutti gli aspetti rilevanti (controllo dei confini, immigrazione, asilo....) e serve a valutare l'evolversi della situazione negli Stati dei Balcani occidentali e ad individuare le azioni da intraprendere. Viste le preoccupazioni degli Stati membri, la Commissione Europea, su invito del Consiglio dei Ministri Europei, ha presentato una proposta per la modifica del Regolamento (CE) n. 539/2001 del Consiglio del 15 marzo 2001 che adotta l'elenco dei Paesi terzi i cui cittadini devono essere in possesso del visto all'atto dell'attraversamento delle frontiere esterne e l'elenco dei Paesi terzi i cui cittadini sono esentati da tale obbligo ${ }^{24}$. Tale proposta di Regolamento prevede l'introduzione di una clausola di salvaguardia che in circostanze eccezionali, permetta di ripristinare temporaneamente l'obbligo di visto per i cittadini di un dato paese terzo. La reintroduzione dell'obbligo di visto non sarebbe automatica, ma a seguito di una valutazione e decisione della Commissione.

Anche se la proposta di modifica Regolamento non è stata ancora adottata, poiché le istituzioni dell'Unione non si stanno raccordando sul testo, una reintroduzione dei visti che implicherebbe un cambiamento nella Regolamentazione dei Visti per l'Europa costituirebbe un passo indietro nelle relazioni con questi paesi, minando anche il processo di liberalizzazione dei visti per altri Stati. Per questo motivo si raccomanda di cercare soluzioni alternative alla reintroduzione dei visti. La stessa Commissione nella sua terza Relazione sul meccanismo di monitoraggio successivo alla liberalizzazione dei visti per i Paesi dei Balcani occidentali sottolinea la necessità di: una maggiore cooperazione con le autorità dei Paesi dei Balcani occidentali; intensificare le indagini su agenzie di viaggio; rafforzare i controlli "in uscita" (cioè nei Paesi dei Balcani occidentali) e "in entrata" (ai confini dell'UE); intensificare campagne informative sui visti per soggiorni di breve durata; aumentare sostanzialmente l'assistenza alle minoranze nei Paesi di origine, in particolare alla comunità rom, che è quella da cui proviene la maggior parte dei richiedenti d'asilo. Nella stessa relazione la Commissione conclude che la maggior parte dei richiedenti asilo è consapevole che la propria domanda di asilo ha poche possibilità di essere accettata, cio significa che tale domande vengono presentate con il solo scopo di rimanere per un certo periodo sul territorio dello Stato che le esamina ottenendo dei benefici, che consistono in vitto e alloggio, assistenza medica ed educazione, mentre alcuni paesi forniscono anche un contributo monetario mensile. Più lunga è la procedura che esamina le richieste, più alti sono dunque i possibili vantaggi per chi richiede asilo pur sapendo di non avere i requisiti per ottenerlo. Proprio nel tempo necessario all'elaborazione delle richieste di asilo che esiste la differenza delle situazioni fra vari paesi, per questo una soluzione del problema sarebbe quella di accelerare l'esame delle domande chiaramente infondate. La legislazione europea permette infatti agli stati membri di dare la priorità alle richieste d'asilo di cittadini provenienti da paesi classificati come "paese d'origine sicuro" (safe country of origin), e di accelerare dunque il processo di esame delle domande. La soluzione migliore sembra quella di adottare un approccio unico europeo al problema, che proponga un limite di tempo di massimo 30 giorni per risolvere le richieste d'asilo di richiedenti che provengono da paesi considerati sicuri, pur salvaguardando i loro diritti come richiedenti.

\section{References}

Manca, L. L'immigrazione del diritto dell'Unione, Milano 2003.

Hailbronner, (2000) "Immigration and Asylum Law and Policy of the European Union", The Hague, London, Boston.

Hailbronner, "Visa Regulation and Third-country Nationals in EC Law", Common Market Law Review, 1994.

Lang, A. "La politica comunitaria in materia di immigrazione", in Diritto Pubblico Comparato ed Europeo, 2003.

Draetta, U. \& Parisi, N. (2010) Elementi di diritto dell'Unione Europea. Giuffré editore.

Bontempi, R. "Gli Accordi di Schengen" in Nascimbene B., (a cura di) "Da Schengen a Maastricht, Apertura delle frontiere, cooperazione giudiziaria e di polizia", Milano 1995.

Liguori, A. L'immigrazione e l'Unione Europea, in Diritto Comunitario e degli Scambi Internazionali, 2000.

Decisione 2007/821/CE del Consiglio, dell'8 novembre 2007. Decisione 2007/822/CE del Consiglio, dell'8 novembre 2007. Decisione 2007/823/CE del Consiglio, dell'8 novembre 2007. Decisione 2007/824/CE del Consiglio, dell'8 novembre 2007. Decisione 2007/825/CE del Consiglio, dell'8 novembre 2007.

COM (79) 115 def. del 27 marzo 1979. 
COM (2011) 290 definitivo

Guce, L 217 del 14 agosto 1985.

Guce C 340 del 10 novembre 1997.

Libro Bianco: II completamento del mercato interno, COM (1985) 310 del 14 giugno 1985

Sentenza della Corte di giustizia del 9 luglio 1987, cause riunite 281, 283, 284, 285 e 287/85,Repubblica federale di Germania, Repubblica francese, Regno dei Paesi Bassi, Regno di Danimarca e Regno Unito di Gran Bretagna e d'Irlanda del Nord c. Commissione delle Comunità europee, in Racc. , 1987.

Regolamento (CE) n. 574/1999 del Consiglio del 12 marzo 1999 che determina quali siano i paesi terzi i cui cittadini devono essere in possesso di un visto per l'attraversamento delle frontiere esterne degli Stati membri in GU L 72 del 18.3.1999.

Regolamento (CE) n. 539/2001 del Consiglio del 15 marzo 2001 che adotta l'elenco dei paesi terzi i cui cittadini devono essere in possesso del visto all'atto dell'attraversamento delle frontiere esterne e l'elenco dei paesi terzi i cui cittadini sono esenti da tale obbligo in GU L 81 del 21.3.2001

Gazzetta ufficiale C 115 del 4.5.2010].Programma di Stoccolma - Un'Europa aperta e sicura al servizio e a tutela dei cittadini www.europa.eu 